\title{
apport des diagraphies pour la réalisation d'injections de carrières effondrées
}

\author{
par \\ J.C. Erling \\ J. Lakshmanan \\ J. Rougé \\ C.P.F.G., Rueil-Malmaison
}

RESUME - La consolidation par injection de carriẽres souterraines plus ou moins effondrées, et de ce fait, inaccessibles, pose des problèmes, à la fois techniques, et surtout financiers. Ce deuxiême aspect devient primordial lorsque les surfaces à injecter sont récluites par ranport à la surface totale des carriēres, ou des cavités karstiques; c'est le cas des tracés linéaires tels que les routes. L'utilisation systématique des diagraphies instantanées - et particuliērement de la vitesse d'avancement et de la percussion réfléchie permet d'assurer la transition entre la reconnaissance de détail et les travaux d'injection.

L'interprétation des enregistrements permet en effet de, ne livrer ã l'injecteur que les trous utiles ã injecter et de mieux prévoir les quantitês et les caractêristiques des coulis à mettre en oeuvre.

En outre, la réalisation de sondages après injection, toujours avec diagraphies, permet de mieux contrôler 1 'efficacité des injections, particulièrement en ce qui concerne le clavage au toit.

Deux exemples illustrent 1'exposé : le premier concerne une voie de desserte d'un groupe d'immeubles en rëgion parisienne, où il s'agissait de Timiter strictement l'extension latérale de l'injection à la zone à consolider. Le deuxième concerne la reprise en sous-oeuvre d'un ouvraçe ayant subi des tassements différentiels notables.

\section{INTRODUCTION}

La recherche d'un programme de confortement optimal nécessite une investigation préalable du terrain par les moyens les plus appropriês. Nous avons mis au point une méthodologie qui permet d'analyser finement par microgravimetrie les variations de T'attraction de la pesanteur et fournit des informations sur les limites des vides dans un plan horizontal. Les sondages destructifs avec diagraphies instantanées (enregistrement de páramẽtres) fournissent des informations précisant les pourcentages de vides dans un plan vertical.

La synthèse des diverses informations alors recueillies permet de fournir un modèle tridimensionnel localisant avec le plus de précision les zones à traiter, et de mettre à disposition de 1 'entreprise chargée des injections les sondages à injecter avec une prêvision des quantités et un planning optimal.

\section{DEFINITION D'UN PROGRAMME DE CONFORTEMENT}

\section{Moyens d'investigation}

Ils ont été décrits en détaị précẻdemment et plus particul ièrement par J. LAKSHMANAN, M. BICHARA et JC. ERLING. (1977).

a) Gravimétrie : La gravimétrie consiste à mesurer les variations de la composante verticale de 1'attraction de la pesanteur. Les carrières souterrajnes et autres vides se manifestent par un déficit de masse qui induit une chute de g fonction de la profondeur et de 1 'importance des vides.

L'appareil utilisé est un gravimètre Lacoste et Romberg, modẽle D, à lecture électronique donnant les mesures relatives avec la précision du microgal (milliardième de l'attraction de la pesanteur). Le maillage doit être adapté au problème posé. Dans le cas de carriēres présentant des zones encore susceptibles d'être visitées, la proportion moyenne de vide est trēs importante. Une maille de $10 \times 10 \mathrm{ri}$ est en général adaptée pourvu que l'extension latérale des carrières soit suffisante (exemples: calcaires lutétiens exploités en région parisienne, carriēres de craie dans le Nord, calcaires bathoniens à Caen).

Dans le cas de vides plus ou moins remblayês au sein d'un ensemble de galeries effondrêes, une maille plus ressérrée $(5 \times 5$ ou même $3 \times 3 \mathrm{~m})$ doit être adoptêe (exemple: exploitations anciennes du tuffeau d'Angers).

Enfin dans le cas d'une structure faillée et karstique recoupant un projet linéaire (par exemple dans les calcaires du Bajocien au Nord de Cluny) une maille relativement resserrée est à adopter, de 1 'ordre de 2 à $5 \mathrm{~m}$ entre mesures.

Dans 1'optique d'une définition d'un programme d'injection, il est nécessaire de faire correspondre aux mesures une géométrie des désordres qui permet de cerner les variations des densités reconstituées à une profondeur définie par la géologie. 


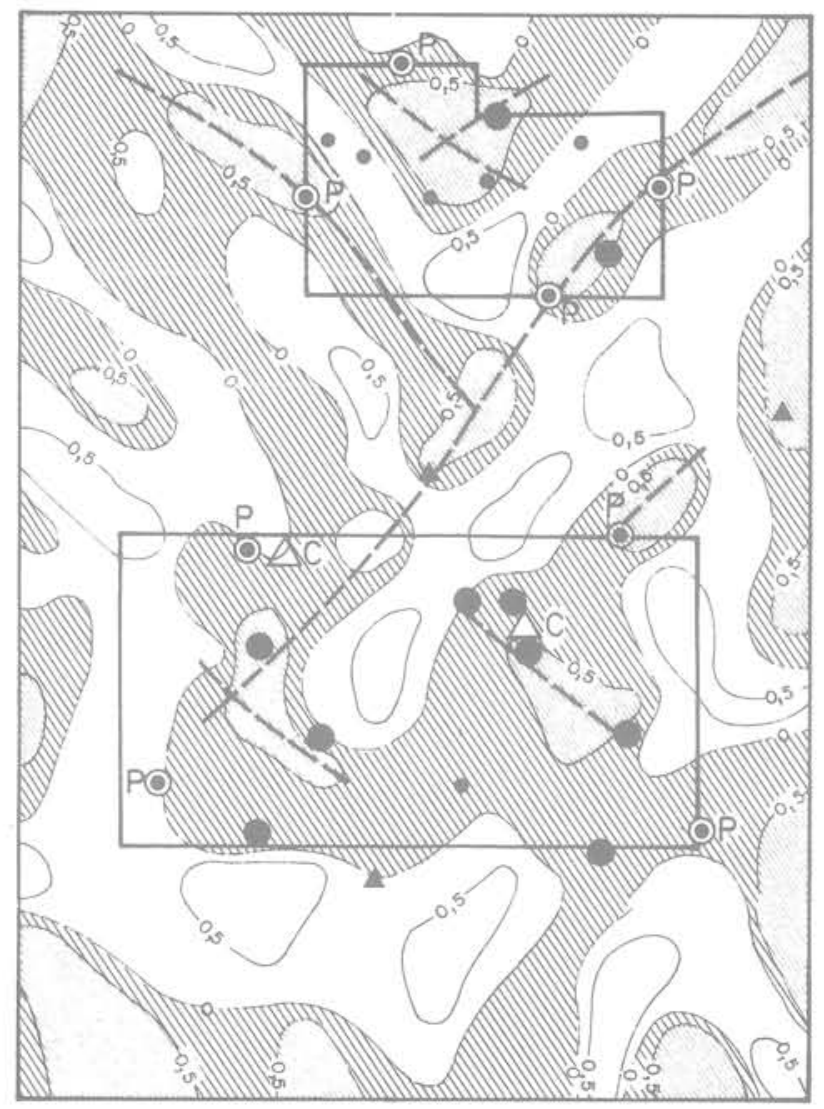

LE LUDE Carte dos densités calculéeset des volumes injectés

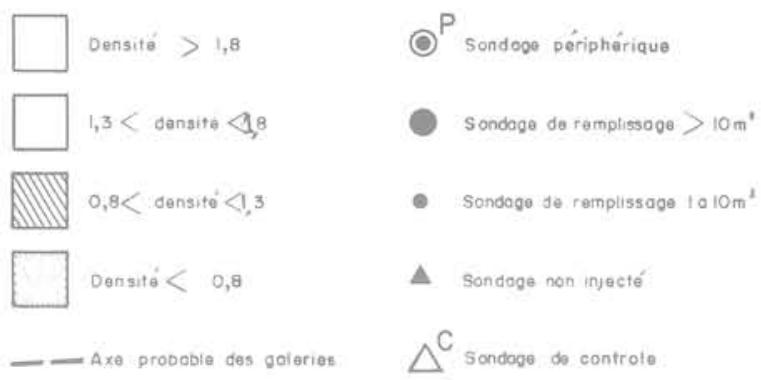

La forme des phénomènes responsables des anomalies est ainsi nettement précisẹe. Ceci nous a en particulier grandement aidé dans la recherche d'un programme d'injections dans le cas de galeries plus ou moins effondrées au Lude.

(voir figure 1).

b) Sondages mécaniques : Pour le contrôle par sondages mécaniques en terrains compacts, le mode de foration le plus adaptē est la roto-percussion à l'air ou à l'eau dans le cas de passages argileux, avec enregistrement instantané de paramètres de forage.

Les deux paramètres les plus adaptés dans ce cas sont la vitesse d'avancement et la percussion réfléchie dans le traín de tige, la vitesse d'avancement étant le paramètre le plus utile pour définir un programme d'injection.

Cependant, l'observation de la seule vitesse d'avancement ne permet pas de discerner entre des vides francs et des vides effondrés remplis d'un matériau peu compact. Cette différence est primordiale en ce sens que des terrains peu combacts neuvent être lạissếs en l'aütat dans la perspective d'une optimisation technico-économique. La percussion réflêchie dans le train de tige permet de différencier les deux types de terrains précités.

Dans un vide franc, $\rceil^{\prime}$ accélaration rêflëchie est très marquée, au contraire $1^{\prime}$ 'on obtient un signal amorti (figures 2 et 3 ) dans un vide remblaye.
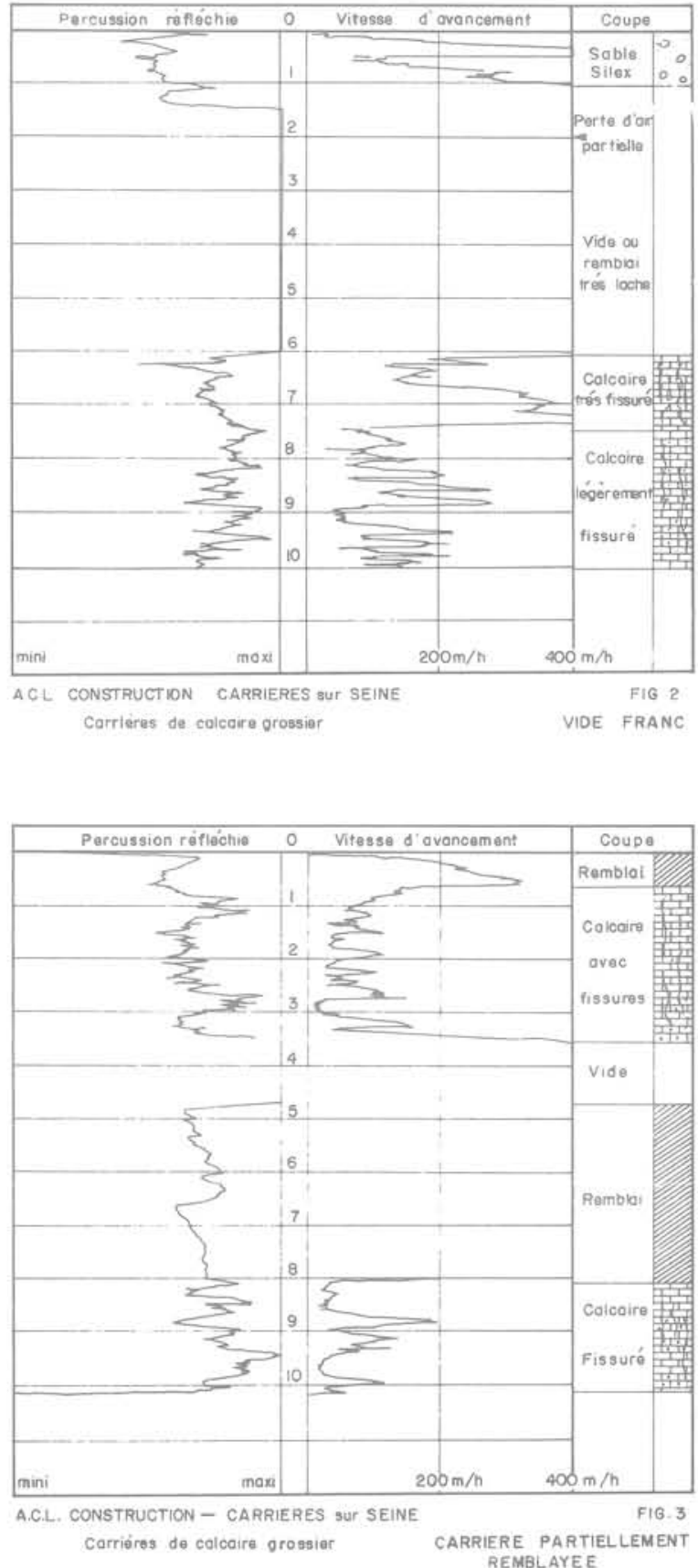
Enfin, il est aisế de distinguer un calcaire très fracturé (figure 4 ) d'un vide remblayé (figure 3 ).

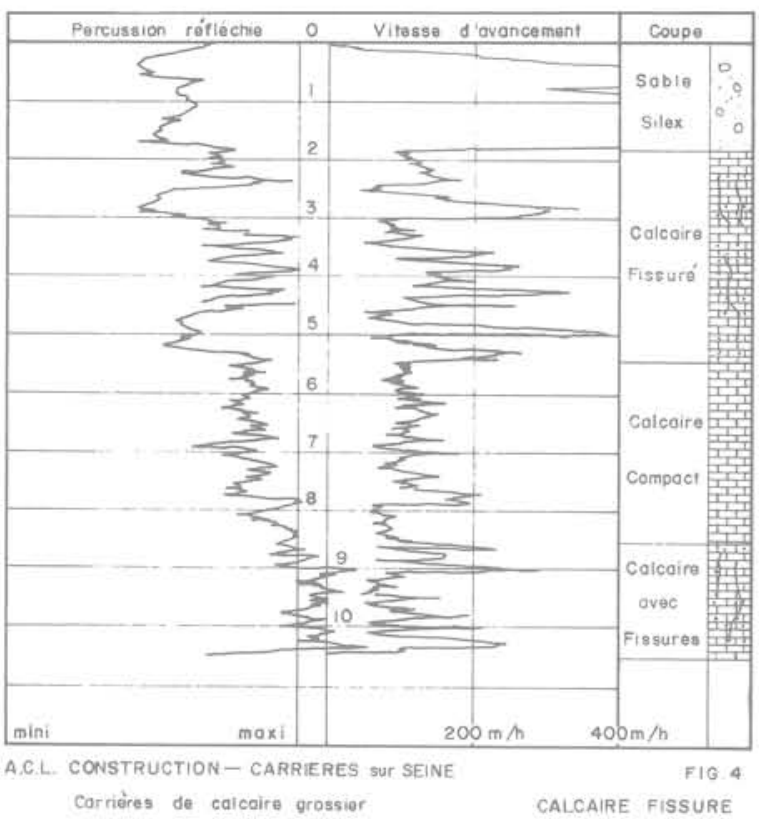

\section{Caractēristiques des injections}

Dans les 2 exemples décrits, il s'agissait d'améliorer les caractéristiques du sol de la façon la plus économique sans pour cela transformer le sol en un "bloc de béton".

\section{a) Choix de la qualité des matériaux à injecter}

Dans tous les cas où T'on craint des extensions latêrales de coulis, il faut définir un programme différent en périphêrie de la zone à traiter.

Dans le cas où les vides sont francs et couvrent à une cote donnée une surface importante, i1 est souhaitable d'édifier des piliers résistants (coulis riche) au sein d'un remplissage initial plus pauvre ou à la limite des piliers seuls.

Dans le cas de structures linéaires à fonder sur des vides étendus, au lieu de prévoir le remplissage hydrautique avec des matériaux économiques maìs ã trẽs faible angle de talus (sablon ou cendres volantes), il convient de prévoir des mélanges comprenant en outre du ciment et de la bentonite.

Dans le cas de cavités souterraines plus ou moins effondrées, il est nécessaire de prévoir une prise rapide du coulis. Pour cela les adjuvants adẹquats (silicate) peuvent être ajoutés au niveau même du forage d'injection, par une pompe doseuse.

\section{b) Choix des pressions d'injections}

Dans les cas semblables à ceux décrits, les pressions d'injections doivent être maintenues faibles en début d'opération et ce n'est que lorsque 1 'on a la certitude de n'avoir aucun départ de coul is hors de la zone à traiter qu'une légère montée en pression peut être tolérée.

\section{c) Choix des niveaux d'injection}

La meilleure façon de procéder pour adapter le programme aux informations des diagraphies est de mettre en oeuvre les quantités injectées par passes successives en insistant au niveau des zones les plus décomprimées. On peut ainsi être amenê â reprendre un forage jusqu'à une dizaine de fois, 1 'espacement dans le temps des passes d'injection augmentant au fur et â mesure des opérations tandis que les quantités injectées sont diminuées dans le même temps.

\section{d) Prévision des quantités à injecter}

La nature des vides à conforter détermine le choix des paramētres à utiliser pour prévoir les quantités à injecter.

D'une manière génêrale, il serait illusoire d'utiliser un seul paramètre pour prévoir les quantitês quelque soit le contexte environnant (extension latórale des fissures, corrélations ou non entre les niveaux dêcomprimés selon les sondages).

Dans le cas de vides francs, la vitesse d'avancement est ã retenir en fixant un seuil très êlevé ( $V>500 \mathrm{~m} / \mathrm{h}$ ou même $V>1000 \mathrm{~m} / \mathrm{h}$ suivant le type de machine de sondages).

Dans le cas de vides remblayés, où T'on peut envisager une légère montée en pression, il convient d'envisager deux types de paramètres :

- hauteur cumulcée de vides présentant une vitesse supérieure à $500 \mathrm{~m} / \mathrm{h}$.

- hauteur cumulẻe de terrains décomprimés présentant des vitesses comprises entre 200 et $500 \mathrm{~m} / \mathrm{h}$.

Dans le cas que nous avons traité au Lude, nous avons હ̃tudiē les corrêlations entre les quantitês injectces et un indice ou "Coefficient de vides" défini pour chaque forage par la formule suivante: $I=\frac{\left(e_{0} I_{0}+e_{1} I_{1}+e_{2} I_{2}+e_{3} I_{3}\right) \times 100}{\left(e_{0}+e_{1}+e_{2}+e_{3}\right)}$

avec $I_{0}=1 \quad$ : vide

$I_{1}=0,6:$ remblai

$I_{2}=0,4$ : terrain décomprimê

$\mathrm{I}_{3}^{2}=0,3$ : calcaire altêré et fissuré

$e_{0}, e_{1}, e_{2}, e_{3}$ : les êpaisseurs correspondantes Si l'on introduit en outre en paramètre la position du forage par rapport à la zone traitée pour tenir compte des variations liêes aux zones non reconnues en périphérie, i† esṫ possible de définir un domaine de corrélation (figure 5).

EXEMPLES

1. Cas de carrières présentant des vides francs et etendus

Dans le cas que nous avons traité à Carrièressur-Seine, pour le compte d'ACL PROMOTION, il 
s'aaissait d'éviter des désordres au droit d'une ćtroite voie de desserte sans injecter les terrains agricoles avoisinants, sous lesquels s'étendaient jusqu'à une grande distance, Tes carriēres de calcaires lutétien. On ne pouvait donc envisager un remplissage systématique à base de sablon ou de cendres volantes. Les vides sont situés entre 5 et $12 \mathrm{~m}$ de profondeur.

Les diagraphies avaient conduit à proposer un optimum technico-financier comprenant des sortes de piliers coniques à travers les vides ou les fontis foisonnés, constitués d'un coulis riche. Dans ce cas T'injection a étહ quasi gravitaire, chaque sondage étant repris entre 2 et 7 fois, avec une injection limitêe à $5 \mathrm{~m}^{3}$ par passe.

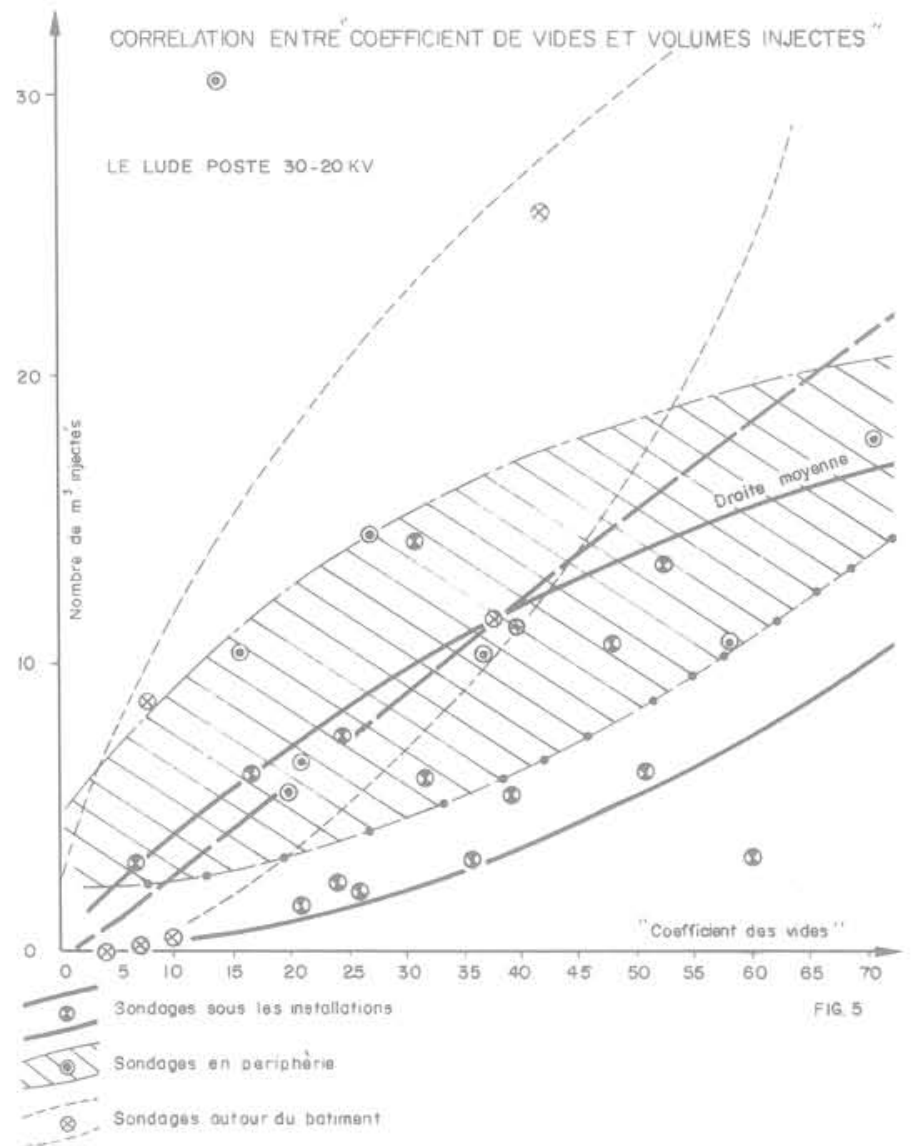

\section{Cas de galeries plus ou moins effondrées}

Dans le cas que nous avons traité au Lude (Sarthe) pour le compte d'Electricité de France, oú il s'agissait de conforter un poste $30 / 20$ KV déjà construit et où 1 'on avait constaté divers désordres, un programme en 2 temps a été défini.Les césordres sont dûs à des galeries creusées dans le tuffeau d'Angers, à moins de $10 \mathrm{~m}$ de profondeur. Il comprenait en premier lieu 1'injection préalable d'un coulis à prise rapide en périphérie des zones présentant des vides. Les matêriaux injectés ont êté placés en piliers au centre des galeries décelées (figure 5).

A $\eta^{\prime}$ intérieur de ce barrage périphêrique, des matêriaux à prise plus lente et donc moins onêreux ont été mis en place.
Dans ce cas une légère montée en pression en fin de colmatage peut être admis, les terrains sêparant les diverses galeries étant susceptibles de se rompre. On peut ainsi injecter des galeries secondaires pouvant être responsables de désordres uttérieurs.

\section{REALISATION D'UN PROGRAIME DE CONTROLE}

\section{Contrôles durant les opêrations d'injection}

Pour avoir une bonne connaissance de 1'évolution du terrain et ëventuellement intervenir en cours d'opération, il convient de vérifier :

- la remontée du coulis sur les forages (figure 6)

- le maintien des pressions d'injection dans les limites fixées

- 1'injection des forages par passes successives et à vitesse d'injection modérée

Si nécessaire, si les matériaux injectés semblent s'écarter de 1 'emprise à conforter, $i 1$ peut être nécessaire de procêder ã :

- une augmentation de la viscositó du coul is

- une diminution de la vitesse de prise

- une diminution du débit d'injection

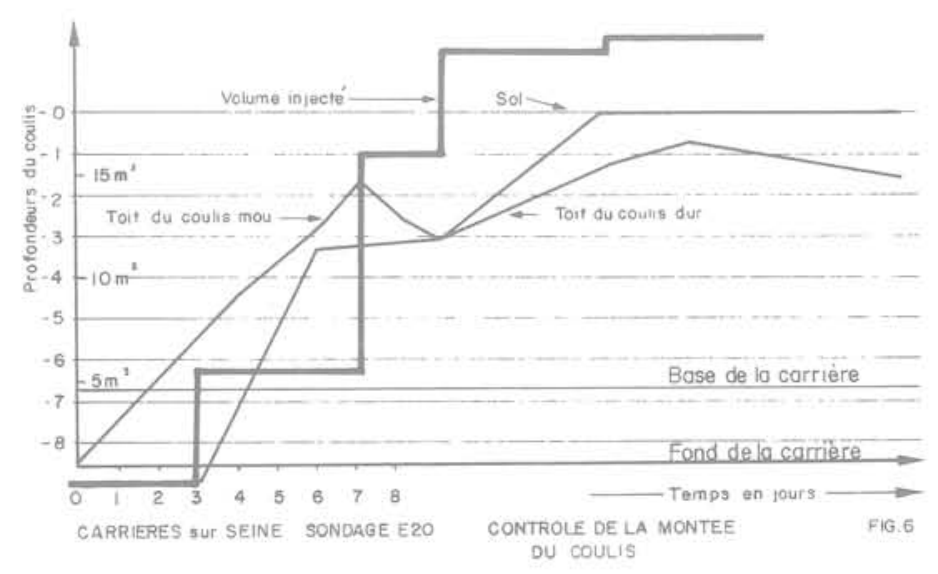

\section{Contrôle après les injections}

Des forages destructifs avec enregistrement de paramètres peuvent être implantós après injection au centre des zones traitées (à une certaine distance des points d'injection). La figure 7 montre 2 enregistrements avant injection et 2 enrecistrements après injection, en des points très proches des 2 premiers, montrant 1 'amé Tioration trēs nette obtenue (chantier du Lude).

\section{CONCLUSION}

Dans les exemples que nous avons évoqués, les résultats des diagraṇhies instantanées ont permis de définir un programme "sur mesures" 1 imitant aux zones à traiter l'extension des matćriaux injectés.

Dans le cas le plus dẹlicat (traitement d'une structure linéaire en zone de vides étendus) les matériaux injectés en dehors de la zone à traiter ne reprêsent pas plus de 10 à $20 \%$ de 1'ensemble. Les prévisions des volumes totales nécessaires à une confortation correcte ont correspondu, à quelcues \% près, aux çuantitiés effectivement mises en oeuvre. 

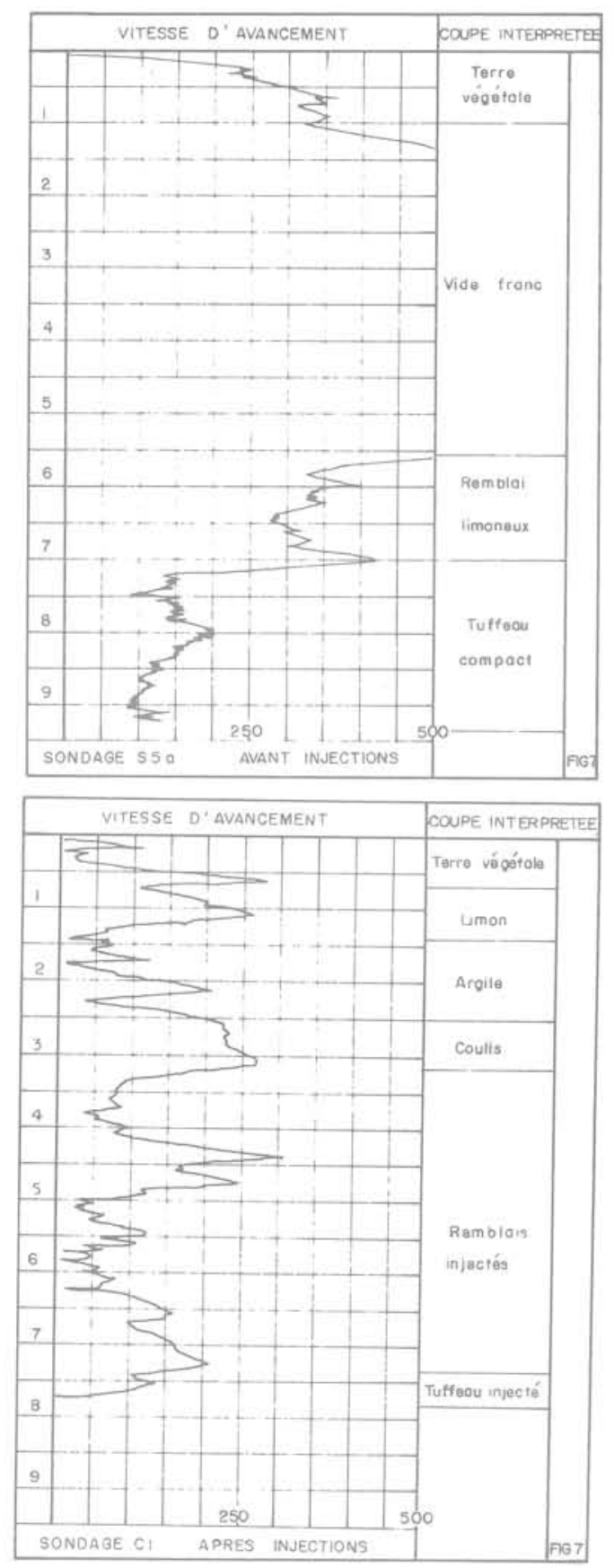

Des canipagnes de remplissage classique auraient conduits à des volumes injectés 5,10 ou 20 fois supérieurs, ce quị, malgré les économies sur les adjuvants, aurait conduit à des dépenses totales plusieurs fois plus grandes.

BIBLIOGRAPHIE

(1) J. LAKSHMANAN, M. BICHARA, JC. ERLING (1977) : Etude de fondation en terrain caverneux, place de la gravimétrie, Bulletin de Liaison, Laboratoire des Ponts et Chaussées - 92 - novembre, décembre 1977.

(2) F. BARDOT, JC. ERLING, L. GIROUSSE, E. SIHERTZ (1979) :

Les problèmes de cavités en milieu urbain,

Colloque National, Lyon, mars 1979 :

Connaitre le sous-so1, un atout pour 1'aménagement urbain.
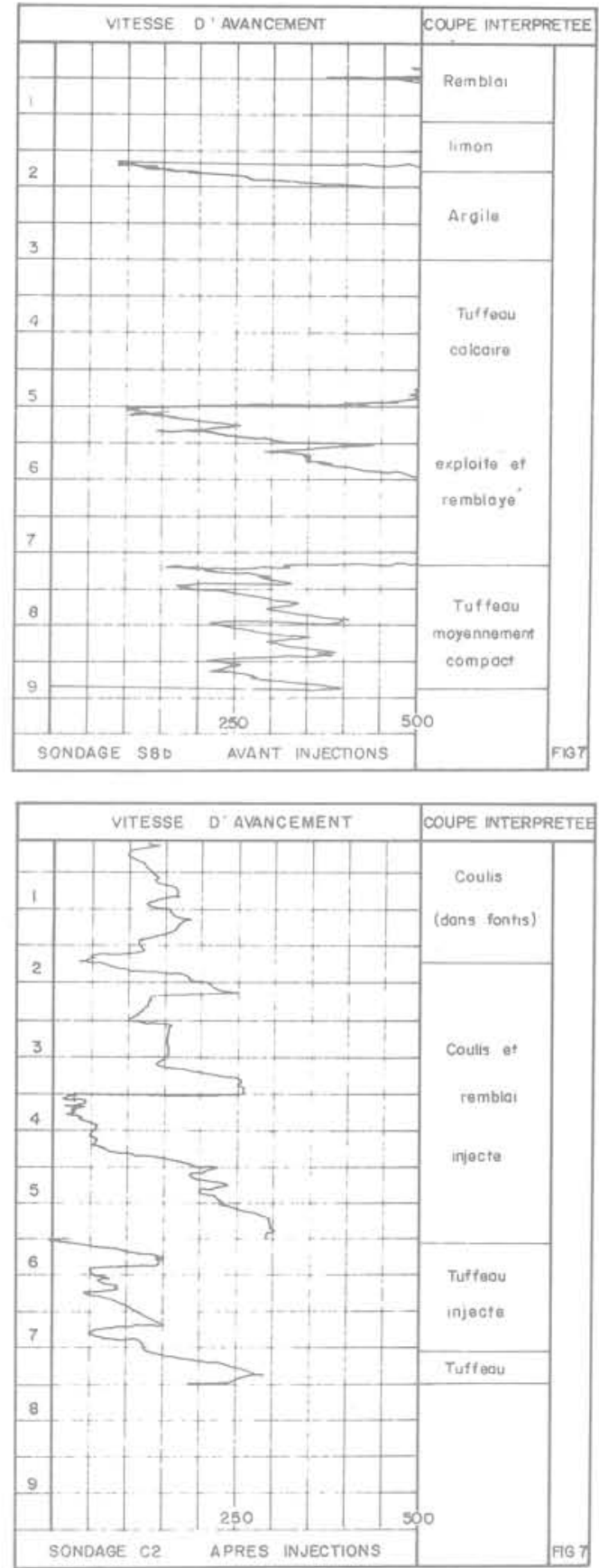

(3) F. RAYMOND, J. LAKSHMANAN, JC, ERLING (1979): Cartographie microgravimétrique de carrières souterraines, application au plan d'aménagement du quartier Est de Caen,

Colloque National, Lyon, mars 1979.

(4) Groupe de Travail prêsidé par M. HABIB, et comprenant notamment $J$. LAKSHMANAN et JC. ERLING (1979) :

Recommandations sur le traitement des cavités souterraines et notamment des carrières (C.S.78), Annales de T'I.T.B.T.P. n 370, mars 1979. 